\title{
COMPUTABILITY AND NONCOMPUTABILITY IN CLASSICAL ANALYSIS
}

BY

\author{
MARIAN BOYKAN POUR-EL AND IAN RICHARDS
}

\begin{abstract}
This paper treats in a systematic way the following question: which basic constructions in real and complex analysis lead from the computable to the noncomputable, and which do not? The topics treated include: computability for $C^{n}, C^{\infty}$, real analytic functions, Fourier series, and Fourier transforms. A final section presents a more general approach via "translation invariant operators". Particular attention is paid to those processes which occur in physical applications. The approach to computability is via the standard notion of recursive function from mathematical logic.
\end{abstract}

This paper represents an excursion through some of the main topics in classical analysis, with a view to determining their computability. We shall move in order through three basic domains: $C^{n}$ functions, analytic functions, and Fourier series and transforms. In a final section, a general approach to several of our results is given via "translation invariant operators." Particular attention will be paid to those operations which are useful in physical applications. Our viewpoint will be that of recursion theory. Thus, as is standard in recursion theory, we make free use of nonconstructive methods to delineate the set of computable functions within the larger set of all functions. In this way, our work differs from that of the intuitionists such as Brouwer and Heyting, the Leningrad school of Sanin, Zaslavskii and others, and the work of Bishop-all of whom are concerned with constructive methods of proof (cf. $[1,2,3,6,16,20])$.

The purpose of this paper is to show which of the basic operations of analysis lead from the computable to the noncomputable, and which do not. Sometimes this is easy to guess. However, there are surprises. Particularly curious, in our view, are certain theorems which give computability in cases where one's first classical intuition would be to look for the "obvious" counterexample. For instance, we would expect that differentiation is not a computable process (because $d / d x$ is not a bounded operator). This is true. But the mere existence of a continuous second derivative of a computable function forces the computability of the first. By extension, of course, if a function $f$ is $C^{\infty}$ (but only $f$ itself is assumed to be computable), then all of its derivatives are computable. One then asks whether the set of derivatives $f, f^{\prime}, f^{\prime \prime}, \ldots, f^{(n)}, \ldots$ is computable as a sequence, uniformly in $n$. Here our intuition is correct: this is too much to ask.

Received by the editors July 17, 1981.

1980 Mathematics Subject Classification. Primary 03D80, 03F60; Secondary 26E05, 26E10, 30E10, 42A20, 42A38, 47A99. 
[It should be remarked that such counterexamples, even when expected, are useful. For they demonstrate that the nonconstructive character of certain traditional proofs is inherent in the theorems themselves. When a noncomputable function is proved to exist from computable data, the proof cannot be constructive.]

We turn now to analytic functions. Here the most natural approach would be to assume the function computable over a region in the complex plane. Then one gets the obvious consequence: by Cauchy's integral formula, the sequence of $n$th derivatives is computable, uniformly in $n$. (Uniformity in $n$ did not hold for $C^{\infty}$ functions; this corresponds to the well-known distinction between " $C^{\infty}$ " and "analytic".) The question becomes more interesting if we assume only that our function is realanalytic and computable along the real line. Then the extension to an open region in the complex plane, while it does exist, does not represent a "well-posed" problem in the sense of Hadamard; that is, very small functions on the real axis can extend to functions which grow arbitrarily rapidly in the $y$-direction in the complex plane. Nevertheless, this question yields a positive answer: if a function $f(x)$ is realanalytic, and if $f$ itself (but not $f^{\prime}, f^{\prime \prime}, \ldots$ ) is assumed computable, then the entire sequence of derivatives is computable, uniformly in $n$. Thus the Taylor series and all related expressions are computable, the Hadamard criterion notwithstanding.

Following the usual progression of classical analysis, we turn next to Fourier series and integrals. Here the difficulties in the classical pointwise theories suggest counterexamples, and this time our intuition turns out to be correct. The counterexamples can be built, following the model of the standard divergent cases. However, the development requires a little care. The most interesting phenomenon, perhaps, is the existence of cases in which there actually is convergence (in fact, uniform convergence), but the convergence is not effective.

All of the above-mentioned results are proved using elementary methods. If we allow more powerful analytic and topological tools, then several of our results can be subsumed into one general theorem, Theorem 7 in $\$ 5$. At the same time, by using a more delicate metrical result which we call the Effective Modulus Lemma, we can strengthen some of our counterexamples (cf. Example 8). We have put this section at the end in order to signal the fact that the earlier results can be handled using only elementary tools from analysis.

So far we have been deliberately vague about the domains on which these various phenomena occur - specifically about the distinction between compact and noncompact domains. We believe that most of the interesting problems occur for compact domains. By contrast, the interplay between logic and analysis, which is the main point of this paper, rarely shows up in the noncompact case. The reason is not far to seek. In the noncompact case, we can construct a sequence of sharp "pulses" and other misbehaviors at will, and thus produce counterexamples. We mention a few of these in passing. The most subtle is Example 5 for entire functions. However, there is one situation where noncompact domains are crucial: the Fourier transform, in which the infinitude of the domain is an essential ingredient of the definition.

The plan of the paper is as follows: One section is devoted to each of the topics mentioned above. Thus we have $\$ 1$ (basic definitions), $\$ 2\left(C^{n}\right.$ functions) and $\$ 3$ 
(analytic functions). In $\$ 4$ we consider the pointwise behavior of Fourier series, Fejer's theorem, and Fourier transforms. The paper concludes with $\$ 5$ (translation invariant operators).

Since the techniques in this paper are drawn from two different areas-recursion theory and analysis-we have presented our arguments in great detail. We believe that they can be followed by interested readers from either discipline. The few exceptions, where we give "sketches", are clearly indicated.

1. Basic definitions and facts. For the sake of reference, we list here the basic definitions and some facts from recursive analysis. The reader is advised to skim this section and return to it when necessary.

We assume as known the idea of a recursive function from the set $\mathbf{N}$ of natural numbers into itself, or from $\mathbf{N}^{q} \rightarrow \mathbf{N}$. Then a sequence $\left\{r_{n}\right\}$ of rational numbers is called computable if there exist three recursive functions $a, b, s$ from $\mathbf{N}$ to $\mathbf{N}$ such that $r_{n}=(-1)^{s(n)}[a(n) / b(n)]$. Similarly we define a computable double or $q$-fold sequence of rationals. We remark that the set of all rationals can be arranged in a computable sequence.

Definition. A real number $x$ is called computable if there is a computable sequence of rationals $\left\{r_{n}\right\}$ which converges effectively to $x$; this means that there is a recursive function $e(n)$ for which $k \geqslant e(n)$ implies $\left|x-r_{k}\right| \leqslant 10^{-n}$. Then, passing effectively to the subsequence $\left\{r_{n}^{\prime}\right\}=\left\{r_{e(n)}\right\}$, we have $\left|x-r_{n}^{\prime}\right| \leqslant 10^{-n}$ for all $n$.

Thus we need two conditions: that the sequence $\left\{r_{n}\right\}$ is computable, and that the convergence is effective. The idea of effective convergence plays a crucial role in our proofs. In cases of noneffective convergence, we cannot guarantee that the limit will be computable, although that might happen by accident. Example 6 and the Effective Modulus Lemma ( $\$ 5)$ show cases in which convergence is noneffective. The limiting value is computable in Example 6, but noncomputable in the Modulus Lemma.

Definition. A sequence of real numbers $\left\{x_{k}\right\}$ is called computable if there is a computable double sequence of rationals $\left\{r_{k n}\right\}$ such that

$$
\left|x_{k}-r_{k n}\right| \leqslant 10^{-n} \text { for all } k, n \text {. }
$$

A vector $\left(x_{1}, \ldots, x_{q}\right) \in \mathbf{R}^{q}$ is called computable if each of its components is a computable real; similarly for sequences of $q$-vectors.

We come now to the notion of a computable function $f: \mathbf{R}^{q} \rightarrow \mathbf{R}^{1}$. This is a central notion in recursive analysis, and we briefly retrace its history. For simplicity, we first consider the case where $f$ is defined on a closed bounded rectangle $I^{q}=\left\{a_{i} \leqslant x_{i} \leqslant b_{i}\right.$, $1 \leqslant i \leqslant q\}$ in $\mathbf{R}^{q}$, whose endpoints $a_{i}, b_{i}$ are computable reals. Banach and Mazur proposed the definition: a function $f: I^{q} \rightarrow \mathbf{R}^{1}$ is computable if it is sequentially computable, i.e.:

(a) $f$ maps every computable sequence of points in $\mathbf{R}^{q}$ into a computable sequence of reals.

This definition was too broad. Grzegorczyk [4] gave a definition based on recursive functionals. He observed that an equivalent definition, in a form usable by 
analysts, can be given by postulating (a) and in addition effective uniform continuity:

(b) There exists a recursive function $d(n)$ such that, for all points $x, y \in I^{q}$,

$$
|x-y| \leqslant 1 / d(n) \text { implies }|f(x)-f(y)| \leqslant 10^{-n} .
$$

Thus we have the well-known $[4,5]$ :

Definition. A function $f: I^{q} \rightarrow \mathbf{R}^{1}$ is computable if it satisfies both conditions (a) and (b) above.

Conditions (a) and (b) have been found useful in recursive analysis because they correspond directly to the classical notions of pointwise evaluation of a function and uniform continuity. In this paper, we find effective uniform continuity playing an especially prominent role in Theorems 1,4 , and 7 , and sequential computability in Example 8.

Other equivalent definitions have been given: cf. Grzegorczyk [4, 5], Lacombe·[9], and Pour-El and Caldwell [12] who give a definition in terms of an effective Weierstrass approximation theorem. The following definition, which uses only mappings on the rationals, was suggested by Mycielski:

A function $f: I^{q} \rightarrow \mathbf{R}$ is computable if and only if there exist recursive functions

$$
\varphi: \mathbf{N} \times Q^{q} \rightarrow Q, \quad \Psi: \mathbf{N} \rightarrow \mathbf{N},
$$

such that

$$
|x-r|<1 / \Psi(n) \text { implies }|f(x)-\varphi(n, r)|<10^{-n},
$$

for $n \in \mathbf{N}, r \in Q^{q}, x \in I^{q}$.

An important extension is the notion of a computable sequence of real-valued functions. Still working over a bounded rectangle $I^{q} \subseteq \mathbf{R}^{q}$, we define

DEFinition. A sequence $\left\{f_{k}\right\}$ of functions $f_{k}: I^{q} \rightarrow \mathbf{R}$ is called computable if

(a) $f_{k}$ maps every computable sequence $\left\{x_{n}\right\}$ of points in $I^{q}$ into a computable double sequence $f_{k}\left(x_{n}\right)$ of reals; and

(b) there exists a recursive function $d(n, k)$ such that, for all points $x, y \in I^{q}$,

$$
|x-y| \leqslant 1 / d(n, k) \text { implies }\left|f_{k}(x)-f_{k}(y)\right| \leqslant 10^{-n} \text {. }
$$

Mycielski's definition can be similarly extended.

REMARK. As an important special case of the above, we observe that if $\left\{f_{k}\right\}$ is a computable sequence of functions, and $x_{0}$ is a fixed computable real, then $\left\{f_{k}\left(x_{0}\right)\right\}$ is a computable (single) sequence of reals. This is used in Example 3.

To extend these notions to the case of functions whose domain is $\mathbf{R}^{q}$, we take the sequence of rectangles $I_{N}=\left\{-N \leqslant x_{i} \leqslant N, 1 \leqslant i \leqslant q\right\}$.

Definition. A function $f: \mathbf{R}^{q} \rightarrow \mathbf{R}^{1}$ is called computable if

(a) $f$ is sequentially computable as above (with $x_{k} \in \mathbf{R}^{q}$ instead of $x_{k} \in I^{q}$ ), and

$\left(\mathrm{b}^{\prime}\right)$ there is a recursive function $d(n, N)$ such that, for all points $x, y$ in the rectangle $I_{N}$,

$$
|x-y| \leqslant 1 / d(n, N) \text { implies }|f(x)-f(y)| \leqslant 10^{-n} \text {. }
$$

The definition of a computable sequence of functions on $\mathbf{R}^{q}$, while similar to the above, is not needed in this paper and will not be spelled out. 
The following are basic results from recursive analysis:

A. If a sequence of functions $\left\{f_{k}\right\}$ is computable, and converges effectively and uniformly to a limit $f$, then $f$ is computable (cf. $[4,5])$.

B. Integration over computable bounded rectangles $I^{q}$ in $\mathbf{R}^{q}$ is a computable process. This is easily established by effectivizing the standard Riemann sum definition.

We conclude with some well-known facts from recursion theory. A set $A$ of natural numbers is called recursively enumerable if there is a recursive function $a(n)$ which enumerates $A$. The function $a(n)$ can be adjusted to be one-to-one. A set $A$ is recursive if both it and its complement in $\mathbf{N}$ are recursively enumerable. There exist recursively enumerable sets which are not recursive. Furthermore, there exist recursively inseparable pairs of sets $A, B$ : this means that $A$ and $B$ are recursively enumerable and disjoint, and there is no recursive set $C$ with $A \subseteq C$ and $B \cap C=\varnothing$.

2. The computable theory of $C^{n}, 1 \leqslant n \leqslant \infty$. Throughout this section, unless stated otherwise, we assume that our functions are defined on a compact interval $[a, b]$ whose endpoints $a, b$ are computable real numbers. (A few results for the infinite interval $(-\infty, \infty)$ are given at the end of the section.) We shall investigate how the notion of computability extends up and down through the various derivatives for $C^{n}$ and $C^{\infty}$.

Integration has already been dispensed with (cf. $\$ 1$ ). Differentiation is considerably trickier. The first result was obtained by Myhill [11], who showed that there exist computable functions which are $C^{1}$ but whose derivatives are not computable. He suggested (in a footnote) that the same should hold for some $C^{\infty}$ functions. However, this is false, for we have

THEOREM 1. If a computable function $f(x)$ on $[a, b]$ is of class $C^{2}$, then its first derivative is computable.

COROLlary 1a. If a computable function $f(x)$ is of class $C^{\infty}$, then each of its derivatives is computable.

On the other hand, Theorem 1 is close to best possible, for by modifying Myhill's counterexample we can show

EXAMPLE 2. There exists a computable function $f(x)$ on $[0,1]$ which is twice differentiable (but not $C^{2}$ ) and whose first derivative is not computable.

There is a corresponding example which delimits Corollary la, namely

EXAMPLE 3. There exists a computable function $f(x)$ on $[-1,1]$ which is $C^{\infty}$, but for which the sequence of derivatives $f(x), f^{\prime}(x), \ldots, f^{(n)}(x), \ldots$ is not computable uniformly in $n$.

We now prove Theorem 1 and Example 3. We have a reason for postponing Example 2; there is a more subtle question which we intend to address below, and it leads to a counterexample which combines nicely with Example 2. This will be covered in the section on "translation invariant operators". 
Proof of TheOrem 1. Recall (cf. $\S 1)$ that $f^{\prime}(x)$ is computable if and only if it is sequentially computable and effectively uniformly continuous. Since $f^{\prime \prime}(x)$ is continuous on the compact interval $[a, b], f^{\prime \prime}(x)$ is bounded, and hence by the mean value theorem $f^{\prime}(x)$ satisfies

$$
\left|f^{\prime}(x)-f^{\prime}(y)\right| \leqslant M \cdot|x-y|, \quad \text { for any integer } M \geqslant \max \left|f^{\prime \prime}(x)\right| .
$$

Thus $f^{\prime}(x)$ is effectively uniformly continuous. We need to prove that it is also sequentially computable. The idea, of course, is to compute $f^{\prime}\left(x_{n}\right)$ as $\lim \left[f(y)-f\left(x_{n}\right)\right] /\left(y-x_{n}\right)$ for some sequence of values $y_{n k} \rightarrow x_{n}$ as $k \rightarrow \infty$. The difficulty is that the difference quotient may not converge effectively. This difficulty vanishes when $f^{\prime}(x)$ is effectively uniformly continuous, as we now show.

Let $\left\{x_{n}\right\}$ be a computable sequence of real numbers, $x_{n} \in[a, b]$. Since $f^{\prime}$ is effectively uniformly continuous, there exists a recursive function $d(n, k)$ such that $\left|y-x_{n}\right| \leqslant 1 / d(n, k)$ implies $\left|f^{\prime}(y)-f^{\prime}\left(x_{n}\right)\right| \leqslant 10^{-k}$. We would like to write $y_{n k}=$ $x_{n}+(1 / d(n, k))$, but this number might be greater than $b$. So we proceed as follows. Since $a, b$, and $\left\{x_{n}\right\}$ are computable, there exist rational numbers $r, s$ and a computable rational sequence $\left\{q_{n}\right\}$ such that

$$
\begin{gathered}
|r-a| \leqslant(b-a) / 10 \\
|s-b| \leqslant(b-a) / 10 \\
\left|q_{n}-x_{n}\right| \leqslant(b-a) / 10 .
\end{gathered}
$$

We can also assume that $1 / d(n, k) \leqslant(b-a) / 10$. Then we define

$$
y_{n k}= \begin{cases}x_{n}+(1 / d(n, k)) & \text { if } q_{n}-r \leqslant(s-r) / 2, \\ x_{n}-(1 / d(n, k)) & \text { otherwise. }\end{cases}
$$

Now we claim that

$$
\left|f^{\prime}\left(x_{n}\right)-\frac{f\left(y_{n k}\right)-f\left(x_{n}\right)}{\left(y_{n k}-x_{n}\right)}\right| \leqslant 10^{-k} .
$$

This follows from the mean value theorem and the definition of $d(n, k)$ : the difference quotient is $f^{\prime}(\xi)$ for some $\xi$ between $x_{n}$ and $y_{n k}$, and $\left|\xi-x_{n}\right| \leqslant 1 / d(n, k)$. Since $f$ is computable, the sequence of difference quotients is computable, uniformly in $n, k$. The error $10^{-k}$ is uniform in $n$, and thus $\left\{f^{\prime}\left(x_{n}\right)\right\}$ is computable.

As mentioned above, we pass over Example 2 (whose proof is given in §5) and proceed to Example 3.

Construction of Example 3. We will build a computable $C^{\infty}$ function $f(x)$ such that the sequence of $n$th derivatives $\left\{f^{(n)}(0)\right\}$ is not bounded by any recursive function.

Take a recursive function $a(n)$ (from $N \rightarrow N$ ) which generates a recursively enumerable nonrecursive set in a one-to-one manner. Assume that $a(n) \neq 0,1$ for all $n$.

Define $R_{n}$ recursively by $R_{0}=1$,

$$
R_{n}=2 n R_{n-1}^{n} \text {. }
$$


(Then $2^{n !} \leqslant R_{n} \leqslant 2^{n^{n}}$ for $n>0$. However, the exact size of $R_{n}$ is not important for our purposes.)

To define our counterexample, set

$$
f(x)=\sum_{k=0}^{\infty} R_{k}^{1-a(k)} \cdot \cos \left(R_{k} x+\frac{\pi}{4}\right), \quad x \in[-1,1] .
$$

This function is clearly computable, since the individual terms of the series are computable, and the series is effectively uniformly convergent (being dominated by $\sum R_{k}^{-1}$ since $a(k) \geqslant 2$; and in view of the fact that $R_{k} \geqslant 2^{k !}$ ).

Furthermore, if $n$ is held fixed, the series for the $n$th derivative is also effectively uniformly convergent: for the series is

$$
f^{(n)}(x)=\sum_{k=0}^{\infty} R_{k}^{n+1-a(k)} \cdot\left\{\begin{array}{c} 
\pm \cos \\
\pm \sin
\end{array}\right\}\left(R_{k} x+\frac{\pi}{4}\right),
$$

and $a(k) \leqslant n+1$ for only finitely many $k$. Then by a well-known theorem of analysis, $f^{(n)}(x)$ exists and is given by this series.

Thus $f \in C^{\infty}$. This means, as we have already proved in Corollary la, that for each fixed $n$, the derivative $f^{(n)}(x)$ is computable. However, we shall show that as $n \rightarrow \infty$, the sequence of values $\left|f^{(n)}(0)\right|$ grows faster than any recursive function. To do this, we use the "pseudo-inverse"

$$
u(n)=\max \{k \mid a(k) \leqslant n\} .
$$

Then $u(n)$ is not recursive; furthermore, there is no recursive function $v(n)$ with $u(n) \leqslant v(n)$. (Otherwise we would have an effective procedure for deciding whether an integer $n$ belongs to the range of $a$.)

Now there is no loss of generality in considering only those values $n$ for which $u(n) \geqslant n$ (infinitely many of these $n$ must exist, since no recursive function bounds $u(n))$. We will show that

$$
\text { if } u(n) \geqslant n \text {, then }\left|f^{(n)}(0)\right| \geqslant u(n) .
$$

Fix a value of $n$ with $u(n) \geqslant n$, and write $m=u(n)$.

We now examine the series given above for $f^{(n)}(x)$. The $m$ th term (which turns out to dominate all of the others) is

$$
R_{m}^{n+1-a(m)} \cdot\{ \pm \sin \text { or } \cos ()\} .
$$

Thus $\mid(m$ th term $)(x=0) \mid$ is $R_{m}^{n+1-a(m)} \cdot \sqrt{1 / 2}$, since the sin or cos is evaluated at $\pi / 4$. Since $a(m) \leqslant n$ (by definition, $m=u(n)=\max \{k \mid a(k) \leqslant n\}$ ), we see that

$$
\mid m \text { th term } \mid \geqslant R_{m} \sqrt{1 / 2} \text {. }
$$

All previous terms, involving $k<m$,

$$
R_{k}^{n+1-a(k)} \cdot\{ \pm \sin \text { or } \cos (\quad)\}
$$

are dominated by $R_{k}^{n} \leqslant R_{k}^{m}$ (since $m=u(n) \geqslant n$, and $a(k) \geqslant 2$ ). Furthermore, there are $m$ such terms. Since $R_{m}=2 m R_{m-1}^{m}$, the sum of these previous terms has absolute value at most $R_{m} / 2$. Since $\sqrt{1 / 2}>1 / 2$ (the difference exceeds 0.1 ), the terms with $k<m$ do not cancel the $m$ th term; in fact the $m$ th term exceeds the sum 
of all previous terms by at least $(0.1) R_{m}>(0.1) 2^{m !}>2 m=2 u(n)$ for $m>2$; thus

$$
\mid \sum_{k=0}^{m}(k \text { th term })(x=0) \mid>2 m=2 u(n), \quad \text { for } u(n) \geqslant n>2 .
$$

As for the terms with $k>m$, these give $a(k)>n$ (again since $m=u(n)=$ $\max \{k \mid a(k) \leqslant n\})$. For at most one value of $k$ is $a(k)=n+1$. Otherwise the exponent $n+1-a(k)$ in

$$
R_{k}^{n+1-a(k)} \cdot\{ \pm \sin \text { or } \cos (\quad)\}
$$

is negative. Since $R_{k} \geqslant 2^{k !}$, the resulting series converges very rapidly to a value which we bound, rather crudely, by 2 . Combining this with $(*)$ we obtain:

For all $n$ where $u(n) \geqslant n>2$,

$$
\left|f^{(n)}(0)\right| \geqslant m=u(n) .
$$

Since $u(n)$ is not bounded by any recursive function, this completes the proof.

We conclude this part with another counterexample, briefly sketched.

REMARK 3a (THE NONCOMPACT CASE). Theorem 1 breaks down if the compact interval $[a, b]$ is replaced by an infinite interval. We shall give the construction but omit any further details, since they are similar to the details in Example 3 and considerably easier. We start with a $C^{\infty}$ function $\varphi(x)$ which is supported on the interval $[-1 / 2,1 / 2]$ and such that its sequence of $n$th derivatives is computable uniformly in $n$; such a function is

$$
\varphi(x)= \begin{cases}e^{-\left[x^{2} /\left(1-4 x^{2}\right)\right]} & \text { for }|x|<1 / 2, \\ 0 & \text { elsewhere. }\end{cases}
$$

Set $\varphi_{n}(x)=(1 / n) \cdot \varphi\left(n^{2} x\right)$, so that $\varphi_{n}$ has amplitude $1 / n$ but $\varphi_{n}^{\prime}$ has amplitude $=$ Const $\cdot n$. Now, as in Example 3, let $a(n)$ be a recursive function which generates a recursively enumerable nonrecursive set in a one-to-one manner. Our counterexample is the function defined on $(-\infty, \infty)$ by

$$
f(x)=\sum_{k=1}^{\infty} \varphi_{k}(x-a(k)) .
$$

Then $f$ is computable over the entire real line, but $f^{\prime}(x)$ grows faster than any computable function as $x \rightarrow+\infty$.

REMARK 3b (SEQUENCES OF FUNCTIONS). Theorem 1 also breaks down if the individual function $f$ is replaced by a computable sequence of functions. The reason is easy to see. For Theorem 1 depended on the compactness of the domain. Now a sequence of functions $\left\{f_{k}(x)\right\}, 0 \leqslant x \leqslant 1$, is the same thing as a single function on the noncompact domain $\mathbf{N} \times[0,1]$. This simple observation allows us to carry over the counterexample of Remark 3a. We merely take the function $f$ on $(-\infty, \infty)$ defined there, and write

$$
f_{k}(x)=f(x+k), \quad 0 \leqslant x \leqslant 1
$$

3. The computable theory of real-analytic functions. Let $f(x)$ be a function which is real-analytic on an open interval $I$ in the real line. (That is, $f$ has a power series 
expansion in some neighborhood of every point $x_{0} \in I$; or equivalently, $f$ has an analytic continuation to some region $\Omega \supset I$ in the complex plane.) This continuation problem is pathological in several respects. For example, as noted in the introduction, the problem is not well posed in the sense of Hadamard: thus the sequence of functions $f_{m}(x)=(1 / m) \cdot \cos m x$, which approaches zero uniformly on the real line, grows arbitrarily rapidly in the imaginary direction as $m \rightarrow \infty$.

Suppose now that $f(x)$ is computable and real-analytic on $I$, but with no assumptions about the computability of its power series or analytic continuation. Surprisingly, in view of the aforementioned pathologies, the continuation problem is well behaved with respect to computability. This we now show.

THEOREM 4. Let $a$ and $r$ be computable real numbers with $0<a<r$. Suppose that $f(x)$ has a Taylor series expansion which converges on $(-r, r)$, and that $f(x)$ is computable on the subinterval $[-a, a]$. Then $f$ has a computable analytic continuation to the disk $\{|z| \leqslant a\}$, and the sequence of derivatives $\left\{f^{(n)}(z)\right\}$ is computable, uniformly in $n$, on this disk.

We note that uniformity in $n$ fails for $C^{\infty}$ functions (Example 3, §2). The theorem also fails for noncompact domains or for sequences of functions (as we will show below). In a sense, these limitations mark the point where the failure of the Hadamard criterion comes into play.

Proof of Theorem 4. Let the Taylor expansion of $f$ be

$$
f(x)=\sum_{k=0}^{\infty} c_{k} x^{k}, \quad-r<x<r .
$$

We have not assumed that the coefficients $c_{n}$ are computable. But by Theorem 1 we know that, since $f$ is computable and $C^{\infty}$ on $[-a, a]$, each of its derivatives is computable; and thus the same holds for each $c_{n}=f^{(n)}(0) / n !$. Our objective is to show that the entire sequence $\left\{c_{n}\right\}$ is computable. To do this, we will use the Taylor series to derive a priori estimates on $\left|f^{(n)}(x)\right|$ which are effective, uniformly in $n$.

From the standard test for radius of convergence,

$$
\limsup _{k \rightarrow \infty} \sqrt[k]{\left|c_{k}\right|} \leqslant 1 / r
$$

Take any computable real number $R$ with $a<R<r$. Then there exists an integer $N_{0}$ such that

$$
k \geqslant N_{0} \text { implies }\left|c_{k}\right|<(1 / R)^{k} \text {. }
$$

Since $N_{0}$ is a fixed integer, the set $\left\{c_{k} \mid k<N_{0}\right\}$ is bounded, and there exists another integer $M_{0}$ such that

$$
\left|c_{k}\right|<M_{0} \text { for } k<N_{0} \text {. }
$$

(Incidentally, it is this choice of $N_{0}, M_{0}$ which does not extend effectively to computable sequences of analytic functions.) We now establish effective bounds on the derivatives $f^{(n)}(z),|z| \leqslant a, n=0,1,2, \ldots$ Differentiating the series for $f$ gives

$$
f^{(n)}(z)=\sum_{k=0}^{\infty} k(k-1) \cdots(k-n+1) c_{k} z^{k-n} .
$$


We bound the first $N_{0}$ terms of this series by

$$
N_{0} \cdot\left[N_{0}^{n} M_{0}(1+a)^{N_{0}}\right] \text {. }
$$

The tail of the series is dominated by

$$
\begin{aligned}
& \sum_{k \geqslant N_{0}} k(k-1) \cdots(k-n+1)\left(a^{k-n} / R^{k}\right) \\
& \leqslant R^{-n} \cdot \sum_{k=0}^{\infty} k(k-1) \cdots(k-n+1)(a / R)^{k-n} .
\end{aligned}
$$

Now this last series is just $R^{-n}(d / d x)^{n}[1 /(1-x)]$ evaluated at $x=a / R<1$. Thus for all $n$ and $|z| \leqslant a$,

$$
\left|f^{(n)}(z)\right| \leqslant N_{0}^{n+1} M_{0}(1+a)^{N_{0}}+R n ![1 /(R-a)]^{n+1} .
$$

This gives us the desired effective bound on the sequence $\left\{f^{(n)}(z)\right\}$.

Now the proof of Theorem 1 can be used to show that the sequence $\left\{f^{(n)}(x)\right\}$ ( $x$ real) is computable, uniformly in $n$. Recall that in Theorem 1, a bound on $f^{\prime \prime}(x)$ gave an effective procedure for computing $f^{\prime}(x)$; and by induction, a bound on $f^{(n)}(x)$ allowed us to compute $f^{(n-1)}(x)$. Now that we have an effective bound on the entire sequence $\left\{\left|f^{(n)}(x)\right|\right\},|x| \leqslant a$, the process of Theorem 1 becomes effective uniformly in $n$. We omit the details.

From the above we see that the sequence of Taylor coefficients $c_{n}=f^{(n)}(0) / n !$ is computable. So, also, are the partial sums of the Taylor series for each $f^{(m)}(z)$. We need to know that these series converge for $\{|z| \leqslant a\}$, effectively in $m$. This follows at once from the methods used above to estimate $\left|f^{(m)}(z)\right|$. Thus the proof is complete.

Theorem 4 does not extend to noncompact domains, as we now show.

EXAMPLE 5 (THE NONCOMPACT CASE FOR ANALYTIC FUNCTIONS). There exists an entire function $f(z)$ whose restriction $f(x)$ to the real line is computable, but such that $f(z)$ is not computable over the whole complex plane, and $f^{\prime}(x)$ is not even computable over the real line.

[Of course, by Theorem $4, f(z)$ is computable on each compact disk $\{|z| \leqslant M\}$.]

SKETCH OF PROOF. The construction is similar to that in Remark $3 a$ above (the noncompact case for $C^{n}$ ), but the verification of its properties is trickier. One difference is that the $C^{\infty}$, compact support function $\varphi(x)$ in Remark 3a is replaced by

$$
\varphi(z)=e^{-z^{2}}
$$

The example is

$$
f(z)=\sum_{k=0}^{\infty} 10^{-k} \varphi\left[10^{2 k}(z-a(k))\right],
$$

where $a(n)$ is as above.

Since the series for $f(x)$ is effectively uniformly convergent on $(-\infty, \infty), f(x)$ is computable on the real line. To prove that $f(z)$ is an entire function, we observe that 
$\varphi(z)=\exp \left(-z^{2}\right)$ satisfies

$$
|\varphi(z)| \leqslant 1 \text { for } 3 \pi / 4 \leqslant \arg (z) \leqslant 5 \pi / 4 .
$$

Now for each fixed $M$, there is an integer $N$ (not a computable function of $M$ ) such that $k>N$ implies $a(k)>100 M$. Hence for $k>N$, the disk $\{|z| \leqslant M\}$ lies entirely within the sector

$$
\{3 \pi / 4 \leqslant \arg (z-a(k)) \leqslant 5 \pi / 4\} .
$$

Thus, after the first $N$ terms, the series for $f(z),|z| \leqslant M$, is dominated by $\Sigma 10^{-k}$, and hence effectively uniformly convergent.

The proof that $f^{\prime}(x)$ is not computable on $(-\infty, \infty)$ follows the pattern of Remark 3a, §2. Finally, since $f^{\prime}(x)$ is not computable on $(-\infty, \infty), f(z)$ cannot be computable over the entire complex plane (otherwise the Cauchy integral formula would give $f^{\prime}(x)$ effectively).

REMARK 5a (SEQUENCES OF FUNCTIONS). Theorem 4 breaks down for computable sequences of real-analytic functions. The construction of the counterexample follows the method of Remark $3 \mathrm{~b}$ above. We take the function $f$ on $(-\infty, \infty)$ given in Example 5, and create a sequence $f_{k}$ by setting $f_{k}(x)=f(x+k)$.

4. The computable theory of Fourier series and transforms. We begin our discussion with Fourier series. Among the questions which naturally present themselves, some have easy answers, and others are subtle. For the sake of completeness, we begin with certain questions which, upon investigation, turn out to be simple. We will dispose of these quickly and then consider the more difficult problems. First we fix some notations.

We consider computable functions $f(x)$ on $[-\pi, \pi]$ such that $f(-\pi)=f(\pi)$. We take the Fourier series in the form

$$
f(x)=\sum_{k=-\infty}^{\infty} c_{k} e^{i k x},
$$

and write $s_{n}(x)$ for the $n$th partial sum

$$
s_{n}(x)=. \sum_{k=-n}^{n} c_{k} e^{i k x} .
$$

Then as is well known,

$$
s_{n}(x)=\frac{1}{2 \pi} \int_{-\pi}^{\pi} f(u) D_{n}(x-u) d u
$$

where $D_{n}(x)$ is the Dirichlet kernel,

$$
D_{n}(x)=\frac{\sin \left(n+\frac{1}{2}\right) x}{\sin \frac{1}{2} x}
$$


We will need estimates on the $L^{1}$-norms of $D_{n}(x)$ and $D_{n}(x) \sin \left(n+\frac{1}{2}\right) x$ (see [7, exercises for Chapter 2.1]):

$$
\begin{aligned}
& \frac{1}{2 \pi} \int_{-\pi}^{\pi}\left|D_{n}(u)\right| d u<999 \log n \text { for } n \geqslant 3, \\
& \frac{1}{2 \pi} \int_{-\pi}^{\pi} \frac{\sin ^{2}\left(n+\frac{1}{2}\right) u}{\left|\sin \frac{1}{2} u\right|} d u>\frac{\log n}{500} \text { for } n \geqslant 1 .
\end{aligned}
$$

Now we return to our investigation. Recall that we have assumed $f(x)$ computable. Then the sequences of Fourier coefficients $\left\{c_{n}\right\}$ and partial sums $\left\{s_{n}(x)\right\}$ are computable, uniformly in $n$; this follows from the standard integral formula for $c_{n}$. So the remaining questions concern the convergence of $\left\{s_{n}(x)\right\}$ to $f(x)$. It is easy to give examples of computable $f$ for which the sequence $\left\{s_{n}(0)\right\}$ does not converge at all; e.g. the counterexample in [19] is a computable function. This leaves the question of whether $\left\{s_{n}(x)\right\}$ can converge uniformly to $f(x)$ without the convergence being effective.

EXAMPLE 6. There exists a computable function $f(x)$ on $[-\pi, \pi]$ with $f(-\pi)=f(\pi)$ such that the Fourier series for $f(x)$ converges uniformly, but the convergence is not effective at the point $x=0$.

Proof. Motivated by the second inequality in $(*)$ above, we start with the computable function

$$
\theta_{n}(x)=\operatorname{sgn} x \cdot \sin \left(n+\frac{1}{2}\right) x,
$$

where sgn $x$ is +1 if $x>0$ and -1 if $x<0$. We define

$$
s_{n, k}(x)=\text { the } k \text { th partial sum in the Fourier series of } \theta_{n}(x) \text {. }
$$

Now we combine the Dirichlet kernel formula with the second and first inequalities in (*) respectively, and use $\left|\theta_{n}\right| \leqslant 1$ to deduce

(1) $s_{n, n}(0)-\theta_{n}(0)>\log n / 1000$ for $\log n>1000$;

(2) $\left|s_{n, k}(x)-\theta_{n}(x)\right|<1000 \log k$ for all $x, n$ and all $k \geqslant 3$.

We need one more formula, which asserts roughly that, although the Fourier series $s_{n, k}(x)$ for $\theta_{n}(x)$ behaves badly when $k$ is near $n$, it quiets down as soon as $k>n^{2}$.

(3) $\left|s_{n, k}(x)-\theta_{n}(x)\right| \leqslant 1000 n / k^{1 / 2}$.

For the sake of completeness, we sketch the proof of (3). Set $E_{k}(x)=\int_{0}^{x} D_{k}(u) d u$. Then $E_{k}(x)$ is bounded uniformly in $k$, and for $|x| \geqslant 1 / k^{1 / 2}, E_{k}(x)$ differs from $\pi \cdot \operatorname{sgn} x$ by less than Const $/ k^{1 / 2}$. Integration by parts in the Dirichlet kernel formula gives

$$
\begin{aligned}
s_{n, k}(x)= & (1 / 2 \pi) \theta_{n}(\pi)\left[E_{k}(x+\pi)-E_{k}(x-\pi)\right] \\
& +(1 / 2 \pi) \int_{-\pi}^{\pi} \theta_{n}^{\prime}(u) E_{k}(x-u) d u .
\end{aligned}
$$

Now everything fits: replacing $E_{k}(x-u)$ by $\pi \cdot \operatorname{sgn}(x-u)$ gives us back $\theta_{n}(x)$; and since $\left|\theta_{n}^{\prime}(u)\right| \leqslant$ Const $\cdot n$, the various errors are dominated by Const $\cdot n / k^{1 / 2}$.

To construct the counterexample $f(x)$, we take, as usual, a recursive function $a(n)$ which enumerates a recursively enumerable nonrecursive set $A$. We define a function 
$R(n)$ which plays roughly the same role as the sequence $R_{n}$ in Example 3:

$$
R(n)=2^{2^{100 n}} \text {. }
$$

Finally we set

$$
f(x)=\sum_{n=0}^{\infty} 2^{-(100 n+a(n))} \boldsymbol{\theta}_{R(n)}(x) .
$$

(In what follows, the reader may note a certain lack of precision in our estimates. We have chosen our parameters so as to minimize the arithmetic.)

The series for $f$ converges effectively and uniformly by comparison with $\Sigma 2^{-100 n}$, since $\left|\theta_{R}\right| \leqslant 1$. Hence $f$ is computable.

Let $s_{k}(x)$ be the $k$ th partial sum in the Fourier expansion of $f(x)$. Then

$$
s_{k}(x)-f(x)=\sum_{n=0}^{\infty} 2^{-(100 n+a(n))}\left[s_{R(n), k}(x)-\theta_{R(n)}(x)\right] .
$$

We break this series into three parts, corresponding to values of $n$ for which

(a) $R(n) \leqslant k^{1 / 4}$,

(b) $k^{1 / 4}<R(n)<k^{C}$ (where $C=2^{50}$ ),

(c) $k^{C} \leqslant R(n)$.

It is important to observe that there is at most one value of $n$ in category (b); for $R(n+1)$ is the $2^{100}$ th power of $R(n)$. We will give upper bounds on (a), (b), and (c) which imply uniform convergence of $s_{k}(x)$ to $f(x)$. Then we will give a lower bound on (b), which shows that in certain cases it is the dominant term: this term does not approach zero effectively. Here are the estimates.

For (a). From (3) above, with $R(n)$ in place of $n$, we get the upper bound

$$
|(\mathrm{a})| \leqslant \sum_{n=0}^{n^{*}} 2^{-100 n}\left[1000 R(n) / k^{1 / 2}\right]
$$

where $n^{*}$ is the largest integer $n$ for which $R(n) \leqslant k^{1 / 4}$. Using this estimate on $R(n)$, together with the fact that $\Sigma 2^{-100 n}<2$, we obtain

$\mid\left(\right.$ a) $\mid \leqslant 2000 / k^{1 / 4}$.

For (b). This time we want both upper and lower bounds. For the former we use (2). There is at most one term in the (b) category, which we estimate by

$\mid$ (b) $\mid \leqslant 2^{-100 n} \cdot 2^{-a(n)}[1000 \log k]$,

where $n$ is the value (if it exists) which puts $R(n)$ in the interval (b). Now $k^{1 / 4}<R(n)$, so $\log k<4 \log R(n)=4 \cdot \log 2 \cdot 2^{100 n}$. Combining this with the above, we get

$|(b)| \leqslant 4000 \cdot 2^{-a(n)}$.

For the lower bound, we consider the special situation where $k=R(n)$ and $x=0$. We use (1). Since $\log R(n)=\log 2 \cdot 2^{100 n}$, we obtain:

If $k=R(n)$ and $x=0$, then (b) $>(1 / 2000) \cdot 2^{-a(n)}$.

For (c). Again we use (2), to obtain

$|(\mathrm{c})| \leqslant \sum_{n=N}^{\infty} 2^{-100 n} \cdot 2^{-a(n)}[1000 \log k]$,

where $N$ is the least integer for which $R(N) \geqslant k^{C}$. Thus $\log k \leqslant(1 / C) \cdot \log R(N)=$ $(1 / C) \cdot \log 2 \cdot 2^{100 N}$. We replace $2^{100 N} \cdot \Sigma_{n \geqslant N} 2^{-100 n}$ by $\sum_{r=0}^{\infty} 2^{-100 r}<2$. Let $a^{*}(N)$ be 
the (noneffective) function

$$
a^{*}(N)=\min _{m \geqslant N} a(m)
$$

Then

$$
|(\mathrm{c})| \leqslant 2^{-a^{*}(N)}(2000 / C) \text {. }
$$

The estimation has finally come to an end. Now we put our results together to give both upper and lower bounds on $\left|s_{k}(x)-f(x)\right|$. These are, respectively,

$$
\left|s_{k}(x)-f(x)\right| \leqslant \frac{2000}{k^{1 / 4}}+4000 \cdot 2^{-a(n)}+2^{-a^{*}(N)}(2000 / C),
$$

where $n$ is the unique integer (if it exists) for which $R(n)$ falls inside the interval (b), $N$ is the smallest integer with $R(N)$ in (c), $C=2^{50}$, and $a^{*}(N)=\min \{a(m) \mid m \geqslant$ $N$ \}. We observe that, when $n$ exists, $N=n+1$.

For the lower bound, we assume that $k=R(n)$ and $x=0$. Then

$$
s_{k}(0)-f(0)>\frac{2^{-a(n)}}{2000}-\frac{2000}{R(n)^{1 / 4}}-2^{-a^{*}(n+1)}(2000 / C),
$$

where $R(n)=2^{2^{100 n}}$.

We note that, from the definition of $R(n)$ and of the intervals (a), (b) and (c), $n$ and $N$ are (up to a constant) on the order of $\log \log k$. Thus $n$ and $N$ approach infinity with $k$. Since $a(n)$ and $a^{*}(N)$ approach infinity, albeit not effectively, the upper bound (A) shows that the Fourier series $s_{k}(x)$ converges uniformly to $f(x)$.

Now we use the lower bound (B) to show that the convergence is not effective at $x=0$. As in Example 3 above, we take the function

$$
u(n)=\max \{k \mid a(k) \leqslant n\} .
$$

Then, as before, $u(n)$ is not bounded above by any recursive function; else the set $A$ would be recursive. Also, we need consider only those $n$ for which $u(n) \geqslant n$. We now reformulate the lower bound in terms of $u(n)$.

Let $m=u(n)$. By definition of $u(n), a(m) \leqslant n$ whereas $a\left(m^{\prime}\right)>n$ for $m^{\prime}>m$. Thus $a^{*}(m+1)>n$ (since $a^{*}(m+1)$ is the minimum of $a\left(m^{\prime}\right)$ for $\left.m^{\prime} \geqslant m+1\right)$. Thus if $k=R(m)$, (B) gives

$$
s_{k}(0)-f(0)>2^{-n}\left[\frac{1}{2000}-\frac{2000}{C}\right]-\frac{2000}{R(m)^{1 / 4}} .
$$

Since $C=2^{50}$, the coefficient of $2^{-n}$ is bounded below by $1 / 3000$. Since $m=u(n)$ $\geqslant n, \quad R(m) \geqslant R(n)$. Also, from the definition of $R(n)$, we trivially deduce $\left(2000 / R(n)^{1 / 4}\right)<\left(2^{-n} / 12,000\right)$ for $n \geqslant 1$. We observe that $(1 / 3000)-(1 / 12,000)$ $=(1 / 4000)$. Putting all of this together, the inequality $(B)$ reduces to the more palatable form:

If $m=u(n), k=R(m)$, then $s_{k}(0)-f(0)>2^{-n} / 4000$.

Now suppose that $s_{k}(0)$ converges effectively to $f(0)$. This means that there is a recursive function $e(r)$ such that

$$
k \geqslant e(r) \text { implies }\left|s_{k}(0)-f(0)\right|<10^{-r} .
$$


Combining this with the previous inequality (and noting that $5^{6}>4000$ ), we have for $r \geqslant 6$,

$$
\begin{aligned}
& k=R(m) \geqslant e(r) \text { implies } n \geqslant r, \text { or } \\
& n<r \text { implies } R(m)=R[u(n)]<e(r) .
\end{aligned}
$$

Since $R[u(n)] \geqslant u(n)$ (with room to spare), this gives an effective upper bound on $u(n)$, which is a contradiction.

REMARK (FEJER'S THEOREM). This asserts that if $f$ is continuous on $[-\pi, \pi]$, $f(-\pi)=f(\pi)$, then the sequence of arithmetic means $\sigma_{n}(x)=(1 /(n+1)) \cdot\left[s_{0}(x)\right.$ $\left.+s_{1}(x)+\cdots+s_{n}(x)\right]$ converges uniformly to $f(x)$. Because it applies to all continuous functions, one would expect Fejer's theorem to effectivize, and it does. By examining any of the standard proofs, we easily verify that if $f$ is computable, then $\sigma_{n}(x)$ converges effectively and uniformly to $f(x)$. In particular, this remark gives an effective version of the Weierstrass approximation theorem for trigonometric polynomials, and hence for ordinary polynomials as well.

Fourier transforms. Since the compact support hypothesis fails, we would hardly expect positive results for Fourier transforms. Following the methods (and notations) of our several previous examples, it can be shown that the function

$$
f(x)=\sum_{k=0}^{\infty} 10^{-(a(k)+k)} e^{-\left(x / 10^{k}\right)^{2}}, \quad x \in \mathbf{R}^{1},
$$

is computable but has a noncomputable Fourier transform.

We would like to do something a little more fundamental. Specialists are well aware that multiplication often behaves badly under the Fourier transform: the theory of multipliers is an outgrowth of this circumstance. Here we show that similar phenomena obtain for computability. Namely, on $\mathbf{R}^{n}(n \geqslant 2)$, we exhibit a case where a computable function $f\left(\omega_{1}, \ldots, \omega_{n}\right)$ has a computable Fourier transform, but $(\cos r) \cdot f, r=\left(\omega_{1}^{2}+\cdots+\omega_{n}^{2}\right)^{1 / 2}$, does not.

In [15], the authors showed that there exist solutions $u\left(x_{1}, \ldots, x_{n}, t\right)$ of the wave equation,

$$
\frac{\partial^{2} u}{\partial x_{1}^{2}}+\cdots+\frac{\partial^{2} u}{\partial x_{n}^{2}}-\frac{\partial^{2} u}{\partial t^{2}}=0 \quad(n \geqslant 2),
$$

which are computable at time $t=0$ but not computable at $t=1$. The initial function $u\left(x_{1}, \ldots, x_{n}, 0\right)$ in [15] is computable and has compact support. Hence its Fourier transform, which involves integration of a computable function over a compact domain, is also computable. Specifically, the Fourier transform is

$$
\hat{u}\left(\omega_{1}, \ldots, \omega_{n}, 0\right)=\int \cdots \int_{\mathbf{R}^{n}} e^{-i\left(\omega_{1} x_{1}+\cdots+\omega_{n} x_{n}\right)} u\left(x_{1}, \ldots, x_{n}, 0\right) d x_{1} \cdots d x_{n}
$$

The wave equation in Fourier transform space becomes

$$
-\left(\omega_{1}^{2}+\cdots+\omega_{n}^{2}\right) \hat{u}-\frac{\partial^{2} \hat{u}}{\partial t^{2}}=0,
$$


where the initial conditions are

$$
\begin{gathered}
\hat{u}\left(\omega_{1}, \ldots, \omega_{n}, 0\right)=\text { the computable Fourier transform given above, } \\
\qquad \frac{\partial \hat{u}}{\partial t}\left(\omega_{1}, \ldots, \omega_{n}, 0\right)=0 .
\end{gathered}
$$

This is a trivial ordinary differential equation in $t$, which we solve to find

$$
\hat{u}\left(\omega_{1}, \ldots, \omega_{n}, t\right)=\hat{u}\left(\omega_{1}, \ldots, \omega_{n}, 0\right) \cdot \cos \left[\left(\omega_{1}^{2}+\cdots+\omega_{n}^{2}\right)^{1 / 2} t\right] .
$$

Clearly $\hat{u}\left(\omega_{1}, \ldots, \omega_{n}, t\right)$, being the product of two computable functions, is computable. In particular, its restriction to the hyperplane $\{t=1\}$ is computable. However, as noted above, the inverse Fourier transform $u\left(x_{1}, \ldots, x_{n}, 1\right)$ is not computable. Since the Fourier transform and its inverse are (modulo a few parameter changes) the same thing, this gives us an effortless way of extracting our counterexample from previous results.

5. Computability for translation invariant operators. Let $X$ and $Y$ be vector spaces of real-valued functions on $\mathbf{R}^{q}$, and let $T: X \rightarrow Y$ be a linear operator such that:

(1) (Translation invariance.) $T$ commutes with convolution, i.e. $T[f * g]=$ $(T f) * g$.

(2) (Compact support.) If $f$ has compact support, then so does $T f$.

(3) (Computability for smooth functions.) Let $\left\{\varphi_{k}\right\}$ be a sequence of $C^{\infty}$-compact support functions $\varphi_{k}: \mathbf{R}^{q} \rightarrow \mathbf{R}^{1}$ for which the multi-sequence of partial derivatives $\left\{\varphi_{k}^{(\alpha)}\right\}$ ( $\alpha=$ multi-index) is computable uniformly in $k$ and $\alpha$. Then the sequence $\left\{T \varphi_{k}\right\}$ is computable.

An example of such an operator is the derivative $d / d x$ on $C^{1}\left[\mathbf{R}^{1}\right]$. In $q$ dimensions, the partial derivatives $\partial / \partial x_{i}$ also have this property. A more recondite example, which occurred in a previous paper of the authors [15], is Kirchhoff's formula for the solution of the wave equation. This formula is a convolution operator which satisfies (1) to (3).

Now we state a general result which holds for operators of this type. In preparation for this, let us recall the two conditions in our working definition of a computable function on a compact domain:

(a) sequential computability;

(b) effective uniform continuity.

These conditions are independent in the sense that, in general, neither implies the other. However, there are situations in which (b) implies (a), and this fact has numerous applications.

THEOREM 7. Let $T$ be a linear operator which satisfies (1)-(3) above. Let $f: \mathbf{R}^{q} \rightarrow \mathbf{R}^{1}$ be a computable function with compact support, and suppose that Tf satisfies condition (b). Then Tf also satisfies (a), i.e. Tf is computable.

As an application of this result, we mention Theorem 1 above. There our proof depended on the fact that, since $f^{\prime \prime}$ was bounded, $T f=(d / d x) f$ was effectively uniformly continuous (condition (b)). Thus Theorem 1 is a corollary of Theorem 7 . (Actually Theorem 1 had slightly different hypotheses, involving the compact 
support of $f$, but that is a detail.) The same principle applies to partial derivatives, and gives an extension of Theorem 1 to several variables.

In our previous work [15] on the wave equation with computable initial conditions, we gave an example in which the solution $u(x, y, z, t)$ was computable at time $t=0$ but not at time $t=1$. In this example, $u(x, y, z, t)$ was a so-called "weak solution": that is, although continuous, it was not twice differentiable. The use of weak solutions is inevitable, and again this is a corollary of Theorem 7. To see this we argue as follows. The transition from the initial condition $f(x, y, z)$ to the solution $u(x, y, z, t)$ is given by an integral operator $T$, called Kirchhoff's operator (cf. [15]). It is trivial to verify that $T$ satisfies our conditions (1)-(3). Now Theorem 7 implies that if $u(x, y, z, 1)$ (setting $t=1$ ) were effectively uniformly continuous, it would be computable. Returning to our original question: if $u(x, y, z, 1)$ were a strong solution (of class $C^{2}$ ), then it would obviously be effectively uniformly continuous. By Theorem 7, it would be computable, and thus could not give the desired counterexample.

Proof of Theorem 7. Because the hypotheses have been carefully formulated, the proof is quite easy. We take any $C^{\infty}$ function $\varphi \geqslant 0$ from $\mathbf{R}^{q} \rightarrow \mathbf{R}^{\mathbf{l}}$ with support on the unit disk in $\mathbf{R}^{q}$, and such that the sequence of derivatives $\left\{\varphi^{(\alpha)}\right\}(\alpha=$ multi-index) is computable. We can suppose that the integral of $\varphi$ over $\mathbf{R}^{q}$ equals 1 . Let $\varphi_{k}(x)=k^{q} \varphi(k x)$, so that the sequence $\left\{\varphi_{k}\right\}$ forms an "approximate identity" for convolution: i.e. the support of $\varphi_{k}$ is a disk of radius $1 / k$ about the origin, and the integral of $\varphi_{k}$ over $\mathbf{R}^{q}$ remains equal to 1 . Now the following fact about convolutions is well known:

Let $g: \mathbf{R}^{q} \rightarrow \mathbf{R}^{1}$ be uniformly continuous, and for $\varepsilon>0$, take $\delta>0$ so that $|x-y| \leqslant \delta$ implies $|g(x)-g(y)| \leqslant \varepsilon$. Then

$$
1 / k \leqslant \delta \text { implies }\left|\left(g * \varphi_{k}\right)-g\right| \leqslant \varepsilon .
$$

To effectivize this, and thus prove Theorem 7, we proceed as follows. Since the operator $T$ satisfies (2) and $f$ has compact support, so does $T f$. Since by (1), $T$ commutes with convolution,

$$
T\left[f * \varphi_{k}\right]=[T f] * \varphi_{k} .
$$

By construction, $\left\{\varphi_{k}^{(\alpha)}\right\}$ is a computable multi-sequence, and since $f$ is computable and integration is a computable process, $\left\{f *\left[\varphi_{k}^{(\alpha)}\right]\right\}$ is computable. Now differentiation also commutes with convolution, and so $f *\left[\varphi_{k}^{(\alpha)}\right]=\left[f * \varphi_{k}\right]^{(\alpha)}$. Hence by (3), $\left\{T\left[f * \varphi_{k}\right]\right\}$ is a computable sequence.

As we have seen, $T\left[f * \varphi_{k}\right]=[T f] * \varphi_{k}$, and this sequence of functions is computable. We will show that $[T f] * \varphi_{k}$ converges effectively and uniformly to $T f$, so that $T f$ is computable. Here we use the assumption that $T f$ satisfies (b) (effective uniform continuity). Thus there is a recursive function $d(n)$ such that $|x-y| \leqslant$ $1 / d(n)$ implies $|T f(x)-T f(y)| \leqslant 10^{-n}$. Now the previously given estimate for convolutions becomes effective, and we have

$$
k \geqslant d(n) \text { implies }\left|\left([T f] * \varphi_{k}\right)-T f\right| \leqslant 10^{-n} .
$$

This completes the proof. 
Having seen several situations in which (b) implies (a), we are naturally led to ask whether (a) also implies (b), so that (a) and (b) are equivalent. The answer is "no". In [15] we gave a counterexample for the wave equation. It was rather intricate, requiring twenty two manuscript pages. Here we give a corresponding example for Theorem 1. Luckily, this new example is much simpler, but it uses many of the same ideas, in particular, the following lemma.

Effective Modulus Lemma. There exists a computable sequence of rational numbers $\left\{r_{m}\right\}$ in $[0,1]$ with the following properties:

(1) the sequence $\left\{r_{m}\right\}$ is strictly increasing and converges to a noncomputable real number $\alpha$;

(2) the differences $\left(r_{m}-r_{m-1}\right)$ do not approach zero effectively;

(3) for any computable sequence of reals $\left\{\gamma_{k}\right\}$, there exist recursive functions $d(k)$ and $e(k)$ such that

$$
\left|\gamma_{k}-r_{m}\right| \geqslant 1 / d(k) \text { for } m \geqslant e(k) .
$$

[ In particular, $\left|\gamma_{k}-\alpha\right| \geqslant 1 / d(k)$ for all $k$.]

We remark that, although there is a uniform procedure which bounds the numbers $\gamma_{k}$ away from $\alpha$, there can be no effective way to determine the signs of all the differences $\left(\gamma_{k}-\alpha\right)$. For if there were, then $\alpha$ would be computable: to see this, we simply take for $\left\{\gamma_{k}\right\}$ any effective enumeration of the rationals.

Before proving the lemma, we show how it applies to the problems discussed in this section.

EXAMPLE 8 (THIS ENCOMPASSES EXAMPLE 2). There exists a computable function $f(x)$ on $[0,1]$ which is twice differentiable (but not $C^{2}$ ) and whose first derivative is sequentially computable but not computable.

[In particular, since $f^{\prime}(x)$ is continuous, this gives an example of a continuous function which is sequentially computable but not computable. Moreover, since $f^{\prime \prime}(x)$ exists, the function $f^{\prime}(x)$ is effectively continuous at each point $x_{0}$, although it is not effectively uniformly continuous. Thus the proof that a continuous function on a compact set is uniformly continuous cannot be effectivized.]

Construction of Example 8 (assuming the Effective Modulus Lemma). Following the notations of the lemma, we set

$$
a_{m}=r_{m}-r_{m-1} \text {. }
$$

Then by (2), the sequence $\left\{a_{m}\right\}$ does not approach zero effectively. Let

$$
b_{m}=\min \left(a_{m}, a_{m-1}\right), \quad m \geqslant 2 .
$$

Since $\left\{r_{m}\right\}$ is strictly increasing, $b_{m}>0$. Finally, we use a computable $C^{\infty}$ pulse function $\varphi(x)$ with support on $[-1 / 2,1 / 2]$, as in Remark 3a near the end of $\S 2$. Now our example is

$$
f(x)=\sum_{m=2}^{\infty} 10^{-m} b_{m} a_{m}^{2} \cdot \varphi\left[10^{m} b_{m}^{-1}\left(x-r_{m-1}\right)\right],
$$

so that

$$
f^{\prime}(x)=\sum_{m=2}^{\infty} a_{m}^{2} \cdot \varphi^{\prime}\left[10^{m} b_{m}^{-1}\left(x-r_{m-1}\right)\right] .
$$


We note that the $m$ th pulse is centered on $r_{m-1}$. The series for $f$ is effectively uniformly convergent by comparison with $\Sigma 10^{-m}$, and it has computable summands, so $f$ is computable. On the other hand, the individual pulses in the series for $f^{\prime}$ have

$$
\begin{aligned}
\text { amplitudes } & =\text { Const } \cdot a_{m}^{2}, \\
\text { half-widths } & =10^{-m} b_{m} \leqslant 10^{-m},
\end{aligned}
$$

where Const $=\max \left|\varphi^{\prime}(x)\right|$. In particular, by definition of $b_{m}$, these pulses do not overlap. Hence $f(x)$ is $C^{\infty}$ except at $\alpha=\lim r_{m}$. But $f^{\prime \prime}(\alpha)$ does exist: $f^{\prime \prime}(\alpha)=0$. To see this, we observe that the $m$ th pulse for $f^{\prime}(x)$ has amplitude $=$ Const $\cdot a_{m}^{2}$ and half-width $\leqslant b_{m} / 2 \leqslant a_{m} / 2$, and that this pulse is centered on $r_{m-1}$. However, $\left(\alpha-r_{m-1}\right)>\left(r_{m}-r_{m-1}\right)=a_{m}$, so that $f^{\prime}(x)$ is squeezed between two parabolas of the form $y= \pm$ Const $\cdot(x-\alpha)^{2}$. Thus $f^{\prime \prime}(\alpha)=0$, and hence $f^{\prime \prime}(x)$ exists at all points.

We now show that $f^{\prime}(x)$, although continuous, is not effectively uniformly continuous and hence not computable. First we recall that the pulses in the series for $f^{\prime}(x)$ have disjoint supports. The $m$ th pulse has height $=$ Const $\cdot a_{m}^{2}$ and half-width $\leqslant 10^{-m}$. Thus the half-widths approach zero effectively, and effective uniform continuity would force the heights to do likewise. But we know that $a_{m}=r_{m}-r_{m-1}$ does not approach zero effectively, and hence neither does $a_{m}^{2}$.

Finally, we show that $f^{\prime}(x)$ is sequentially computable, i.e. that the sequence $\left\{f^{\prime}\left(\gamma_{k}\right)\right\}$ is computable for any computable sequence of reals $\left\{\gamma_{k}\right\}$. It is here that we need the Effective Modulus Lemma. For the series which gives $f^{\prime}(x)$ does not converge effectively (it cannot, since $f^{\prime}(x)$ is not computable), and to sum this series at $x=\gamma_{k}$ requires special considerations.

Take any computable sequence of reals $\left\{\gamma_{k}\right\}$; by the lemma there exist recursive functions $d(k)$ and $e(k)$ such that

$$
\left|\gamma_{k}-r_{m}\right| \geqslant 1 / d(k) \text { for } m \geqslant e(k) .
$$

Using this information, we can sum the series for $f^{\prime}\left(\gamma_{k}\right)$ in a finite number of steps, by a procedure which is uniform in $k$. Namely, let

$$
M(k)=\max (m(k), e(k)),
$$

where

$$
m(k)=\text { the least integer with } 10^{-m(k)} \leqslant 1 / d(k) .
$$

Then to compute $f^{\prime}\left(\gamma_{k}\right)$, we simply compute the first $M(k)$ terms in the series for $f^{\prime}(x)$; all other terms vanish at $x=\gamma_{k}$. To see this, we recall that the $m$ th pulse is centered on $r_{m-1}$ and has half-width $\leqslant 10^{-m}$. When $m>M(k)$, then

$$
10^{-m}<10^{-m(k)} \leqslant 1 / d(k)
$$

but $m>e(k)$ and so

$$
\left|\gamma_{k}-r_{m-1}\right| \geqslant 1 / d(k),
$$

whence the $m$ th pulse does not reach the point $x=\gamma_{k}$. This completes the proof. 
Proof of the Effective Modulus Lemma. This was proved in [15], but for the sake of completeness we give an abbreviated version here. Our starting point is a pair of recursively inseparable sets $A$ and $B$; that is, the sets $A, B$ are recursively enumerable and disjoint, and there is no recursive set $C$ with $A \subseteq C$ and $B \cap C=\varnothing$. Let $a(n)$ and $b(n)$ be recursive functions which enumerate $A$ and $B$ in a one-to-one fashion. Now we set

$$
r_{m}=\frac{5}{9}+\sum_{n=0}^{m} 10^{-a(n)}, \quad \alpha=\frac{5}{9}+\sum_{n=0}^{\infty} 10^{-a(n)}
$$

Thus the decimal expansion of $\alpha$ is a sequence of 5's and 6's, with a 6 in the $s$ th place if and only if $s \in A$. (We can assume that $0 \notin A$, so that $0<\alpha<1$.)

Now take any computable sequence of reals $\left\{\gamma_{k}\right\}$. We will want to consider the decimal expansions of the $\gamma_{k}$, and there is the difficulty that these expansions may not be effectively determined, because of the ambiguity between decimals ending in $000 \ldots$ versus $999 \ldots$ However, we can construct a computable double sequence of "finite decimal approximations" to the $\gamma_{k}$, using the following steps:

Since $\left\{\gamma_{k}\right\}$ is computable (cf. $\S 1$ ), there exists a computable double sequence of rationals $\left\{R_{k, q}\right\}$ such that $\left|R_{k, q}-\gamma_{k}\right| \leqslant 10^{-(q+2)}$. Of course, the decimal expansion of a rational number is computable. We define the ( $q$ th decimal for $\gamma_{k}$ ) to be that decimal of length $q+1$ which most closely approximates $R_{k, q}$; in case of ties we take the smaller one. Then $\mid\left(q\right.$ th decimal for $\left.\gamma_{k}\right)-R_{k q} \mid \leqslant(1 / 2) 10^{-(q+1)}$ and $\left|R_{k q}-\gamma_{k}\right| \leqslant(1 / 10) 10^{-(q+1)}$, so that

$$
\mid\left(q \text { th decimal for } \gamma_{k}\right)-\gamma_{k} \mid \leqslant 10^{-(q+1)} \text {. }
$$

Then the double sequence ( $q$ th decimal for $\gamma_{k}$ ) is computable in the sense that, if we write

$$
\left(q \text { th decimal for } \gamma_{k}\right)=N_{q, 0} \cdot N_{q, 1} N_{q, 2} \cdots N_{q, q+1}
$$

then $N_{q, s}=N_{q, s}(k)$ (where $s \leqslant q+1$ ) is recursive in $q, s$, and $k$. This is the desired effective sequence of finite decimal approximations to $\gamma_{k}$.

Now we come to the heart of the proof. It is here that we use the other set $B$ in our recursively inseparable pair. Consider any particular $\gamma_{k}$. We list the sets $A$ and $B$ in turn, using the recursive functions $a(n)$ and $b(n)$. We stop when we come to an integer $s \in A \cup B$ such that either:

(a) $s \in A$ and $N_{s, s} \neq 6, N_{s, s+1} \neq 0$ or 9 , or

(b) $s \in B$ and $N_{s, s}=6, N_{s, s+1} \neq 0$ or 9 , or

(c) $s \in A \cup B$ and $N_{s, s+1}=0$ or 9 .

One of the situations (a), (b), or (c) must eventually occur. To prove this, suppose that (c) never occurs. Then let $C$ denote the set of integers $\left\{s \mid N_{s, s}=6\right\}$. Since the function $N_{s, s}(k)$ is recursive, $C$ is recursive, uniformly in $k$. Hence, since the sets $A$ and $B$ are recursively inseparable, we cannot have $A \subseteq C$ and $B \subseteq \bar{C}$. If $A \nsubseteq C$ then 
we have (a), and if $B \nsubseteq \bar{C}$ then we have (b). Furthermore, this procedure can be carried out uniformly in $k$, by an effective process which returns to each $\gamma_{k}$ infinitely often.

Now we define the functions $d(k)$ and $e(k)$ of the lemma. We let $s$ be the first occurrence of a value $s=a(n)$ or $s=b(n)$ for which (a), (b), or (c) holds. Then we set

$$
d(k)=10^{s+1}, \quad e(k)=n .
$$

We must verify that $\left|r_{m}-\gamma_{k}\right| \geqslant 1 / d(k)$ for $m \geqslant e(k)$.

Recall that the $s$ th decimal digit for $\alpha$ is a 6 if $s \in A$, and otherwise it is a 5 . Furthermore, since $a(n)$ gives a one-to-one enumeration of $A$, and $A \cap B=\varnothing$, the $s$ th decimal digit for $r_{m}$ is determined as soon as some $a(n)$ or $b(n)$ equals $s$. By definition of $e(k)$, this occurs as soon as $m \geqslant e(k)$. Then the $s$ th digit for $r_{m}$ coincides with that for $\alpha$ (6 if $s \in A$, otherwise 5).

Now, taking $m \geqslant e(k)$, we show that $\left|r_{m}-\gamma_{k}\right| \geqslant 10^{-(s+1)}=1 / d(k)$. The proof depends on whether the above process terminated in (a), (b), or (c). If (c), then the $(s+1)$ st decimal digit for $\gamma_{k}$ is $8,9,0$, or 1 (allowing for errors in the decimal approximation), whereas the $(s+1)$ st digit for $r_{m}$ is 5 or 6 . This gives a discrepancy $\geqslant 10^{-(s+1)}$, as desired. If (a), then the $s$ th digit for $r_{m}$ is 6 , whereas the $s$ th digit for $\gamma_{k}$ is not 6 ; here we use the fact that $N_{s, s+1} \neq 0$ or 9 . Again we have a discrepancy $\geqslant 10^{-(s+1)}$. The case (b) is handled similarly, and this completes the proof.

\section{REFERENCES}

1. O. Aberth, Computable analysis, McGraw-Hill, New York, 1980.

2. E. Bishop, Foundations of constructive analysis, McGraw-Hill, New York, 1967.

3. L. E. J. Brouwer, De Onbetrouwbaarheid der Logische Principes (the untrustworthiness of the principles of logic), Tijdschrift voor Wijsbegeerte, Amsterdam, vol. 2, 1908, 152-158.

4. A. Grzegorczyk, Computable functionals, Fund. Math. 42 (1955), 168-202.

5. On the definitions of computable real continuons functions, Fund. Math. 44 (1957), 61-71.

6. A. Heyting, Intuitionism, an introduction, studies in logic and foundations of mathematics, North-Holland, Amsterdam, 1966.

7. Y. Katznelson, An introduction to harmonic analysis, Wiley, New York, 1968.

8. G. Kreisel, A notion of mechanistic theory, Synthese 29 (1974), 11-16.

9. D. Lacombe, Extension de la notion de fonction récursive aux fonctions d' une ou plusieurs variables réelles. I, II, III, C. R. Acad. Sci. Paris 240 (1955), 2478-2480; 241 (1955), 13-14; 241 (1955), 151-153.

10. Y. N. Moschovakis, Notation systems and recursive ordered fields, Compositio Math. 17 (1965), 40-71.

11. J. Myhill, A recursive function defined on a compact interval and having a continuous derivative that is not recursive, Michigan Math. J. 18 (1971), 97-98.

12. M. B. Pour-El and J. Caldwell, On a simple definition of computable function of a real variable-with applications to functions of a complex variable, Z. Math. Logik. Grundlagen Math. 21 (1975), 1-19.

13. M. B. Pour-El and I. Richards, Differentiability properties of computable functions-a summary, Acta Cybernet. 4 (1978), 123-125.

14. __ A computable ordinary differential equation which possesses no computable solution, Ann. Math. Logic 17 (1979), 61-90.

15. The wave equation with computable initial data such that its unique solution is not computable, Adv. in Math. 39 (1981), 215-239.

16. N. A. Šanin, Constructive real numbers and constructive function spaces, Transl. Math. Monographs, vol. 21, Amer. Math. Soc., Providence, R. I., 1968. 
17. J. C. Shepherdson, On the definition of computable function of a real variable, Z. Math. Logik. Grundlagen Math. 22 (1976), 391-402.,

18. E. Specker, Der Satz vom Maximum in der rekursiven Analysis, Constructivity in Mathematics, Proc. Amsterdam Colloq. 1957 (A. Heyting, ed.), Studies in Logic and Foundations of Math., North-Holland, Amsterdam, 1959.

19. E. C. Titchmarsh, The theory of functions, Oxford Univ. Press, New York, 1939.

20. I. D. Zaslavskii, Some properties of constructive real numbers and constructive functions, Amer. Math. Soc. Transl. (2) 57 (1966), 1-84.

SChOOL of Mathematics, University of Minnesota, Minneapolis, Minnesota 55455 